\title{
Motivic Milnor Fibers and Jordan Normal Forms of Milnor Monodromies
}

\author{
by \\ Yutaka Matsui and Kiyoshi TAKeUCHI
}

\begin{abstract}
By calculating the equivariant mixed Hodge numbers of motivic Milnor fibers introduced by Denef-Loeser, we obtain explicit formulas for the Jordan normal forms of Milnor monodromies. The numbers of the Jordan blocks will be described by the Newton polyhedron of the polynomial.

2010 Mathematics Subject Classification: Primary 32S35; Secondary 14E18, 14M25, 32C38, 32S40.

Keywords: Milnor fiber, Milnor monodromy, mixed Hodge number, Newton polyhedron.
\end{abstract}

\section{$\S 1$. Introduction}

In this paper, by using motivic Milnor fibers introduced by Denef-Loeser [4], [5], we obtain explicit formulas for the Jordan normal forms of Milnor monodromies. Let $f(x)=\sum_{v \in \mathbb{Z}_{+}^{n}} a_{v} x^{v} \in \mathbb{C}\left[x_{1}, \ldots, x_{n}\right]$ be a polynomial on $\mathbb{C}^{n}$ such that the hypersurface $f^{-1}(0)=\left\{x \in \mathbb{C}^{n} \mid f(x)=0\right\}$ has an isolated singular point at $0 \in \mathbb{C}^{n}$. Then by a fundamental theorem of Milnor [18], the Milnor fiber $F_{0}$ of $f$ at $0 \in \mathbb{C}^{n}$ has the homotopy type of a bouquet of $(n-1)$-spheres. In particular, we have $H^{j}\left(F_{0} ; \mathbb{C}\right) \simeq 0(j \neq 0, n-1)$. Denote by

$$
\Phi_{n-1,0}: H^{n-1}\left(F_{0} ; \mathbb{C}\right) \stackrel{\sim}{\longrightarrow} H^{n-1}\left(F_{0} ; \mathbb{C}\right)
$$

the $(n-1)$-th Milnor monodromy of $f$ at $0 \in \mathbb{C}^{n}$. By the theory of monodromy zeta functions due to A'Campo [1], Varchenko [27] and others, the eigenvalues of $\Phi_{n-1,0}$ are fairly well-understood. See Oka's book [20] for an excellent exposition.

Communicated by T. Mochizuki. Received June 5, 2013. Revised October 13, 2013.

Y. Matsui: Department of Mathematics, Kinki University, 3-4-1, Kowakae, Higashi-Osaka, Osaka, 577-8502, Japan;

e-mail: matsui@math.kindai.ac.jp

K. Takeuchi: Institute of Mathematics, University of Tsukuba, 1-1-1, Tennodai, Tsukuba, Ibaraki, 305-8571, Japan;

e-mail: takemicro@nifty.com

(C) 2014 Research Institute for Mathematical Sciences, Kyoto University. All rights reserved. 
However to the best of our knowledge, it seems that the Jordan normal form of $\Phi_{n-1,0}$ is not fully understood yet. In this paper, we give a combinatorial description of the Jordan normal form of $\Phi_{n-1,0}$ by using motivic Milnor fibers (for a computer algorithm via Brieskorn lattices, see e.g. Schulze [24]) in the case where $f$ is convenient and non-degenerate at $0 \in \mathbb{C}^{n}$ (see Definitions 4.1 and 4.2).

From now on, let us assume also that $f$ is convenient and non-degenerate at $0 \in \mathbb{C}^{n}$. Note that the second condition is satisfied by generic polynomials $f$, if the Newton polyhedron is fixed. Then we can describe the Jordan normal form of $\Phi_{n-1,0}$ very explicitly as follows. We call the convex hull of $\bigcup_{v \in \operatorname{supp}(f)}\left\{v+\mathbb{R}_{+}^{n}\right\}$ in $\mathbb{R}_{+}^{n}$ the Newton polyhedron of $f$ and denote it by $\Gamma_{+}(f)$. Let $q_{1}, \ldots, q_{l}$ (resp. $\gamma_{1}, \ldots, \gamma_{l^{\prime}}$ ) be the 0 -dimensional (resp. 1-dimensional) faces of $\Gamma_{+}(f)$ such that $q_{i} \in \operatorname{Int}\left(\mathbb{R}_{+}^{n}\right)$ (resp. the relative interior rel.int $\left(\gamma_{i}\right)$ of $\gamma_{i}$ is contained $\left.\operatorname{in} \operatorname{Int}\left(\mathbb{R}_{+}^{n}\right)\right)$. For each $q_{i}\left(\right.$ resp. $\left.\gamma_{i}\right)$, denote by $d_{i}>0$ (resp. $\left.e_{i}>0\right)$ its lattice distance $\operatorname{dist}\left(q_{i}, 0\right)$ (resp. $\left.\operatorname{dist}\left(\gamma_{i}, 0\right)\right)$ from the origin $0 \in \mathbb{R}^{n}$. For $1 \leq i \leq l^{\prime}$, let $\Delta_{i}$ be the convex hull of $\{0\} \sqcup \gamma_{i}$ in $\mathbb{R}^{n}$. Then for $\lambda \in \mathbb{C} \backslash\{1\}$ and $1 \leq i \leq l^{\prime}$ such that $\lambda^{e_{i}}=1$ we set

$$
\begin{aligned}
n(\lambda)_{i}= & \sharp\left\{v \in \mathbb{Z}^{n} \cap \operatorname{rel} \operatorname{int}\left(\Delta_{i}\right) \mid \operatorname{ht}\left(v, \gamma_{i}\right)=k\right\} \\
& +\sharp\left\{v \in \mathbb{Z}^{n} \cap \operatorname{rel} \operatorname{int}\left(\Delta_{i}\right) \mid \operatorname{ht}\left(v, \gamma_{i}\right)=e_{i}-k\right\},
\end{aligned}
$$

where $k$ is the minimal positive integer satisfying $\lambda=\zeta_{e_{i}}^{k}\left(\zeta_{e_{i}}:=\exp \left(2 \pi \sqrt{-1} / e_{i}\right)\right)$ and for $v \in \mathbb{Z}^{n} \cap$ rel.int $\left(\Delta_{i}\right)$ we denote by $\operatorname{ht}\left(v, \gamma_{i}\right)$ the lattice height of $v$ from the base $\gamma_{i}$ of $\Delta_{i}$. Then in Section 4 we prove the following result. Recall that by the monodromy theorem the sizes of such Jordan blocks are bounded by $n$.

Theorem 1.1. Assume that $f$ is convenient and non-degenerate at $0 \in \mathbb{C}^{n}$. Then for any $\lambda \in \mathbb{C}^{*} \backslash\{1\}$ we have:

(i) The number of Jordan blocks for the eigenvalue $\lambda$ with the maximal possible size $n$ in $\Phi_{n-1,0}: H^{n-1}\left(F_{0} ; \mathbb{C}\right) \stackrel{\sim}{\longrightarrow} H^{n-1}\left(F_{0} ; \mathbb{C}\right)$ is equal to $\sharp\left\{q_{i} \mid \lambda^{d_{i}}=1\right\}$.

(ii) The number of Jordan blocks for the eigenvalue $\lambda$ with size $n-1$ in $\Phi_{n-1,0}$ is equal to $\sum_{i: \lambda^{e_{i}=1}} n(\lambda)_{i}$.

For the results concerning lower sizes, see Theorem 4.6. Namely the Jordan blocks for the eigenvalues $\lambda \neq 1$ in the monodromy $\Phi_{n-1,0}$ are determined by the lattice distances of the faces of $\Gamma_{+}(f)$ from the origin $0 \in \mathbb{R}^{n}$. The monodromy theorem asserts also that the sizes of the Jordan blocks for the eigenvalue 1 in $\Phi_{n-1,0}$ are bounded by $n-1$. In this case, we have the following result. Denote by $\Pi_{f}$ the number of lattice points on the 1 -skeleton of $\partial \Gamma_{+}(f) \cap \operatorname{Int}\left(\mathbb{R}_{+}^{n}\right)$. For a compact face $\gamma \prec \Gamma_{+}(f)$, denote by $l^{*}(\gamma)$ the number of lattice points on the relative interior rel.int $(\gamma)$ of $\gamma$. 
Theorem 1.2. In the situation of Theorem 1.1 we have:

(i) (van Doorn-Steenbrink [6]) The number of Jordan blocks for the eigenvalue 1 with the maximal possible size $n-1$ in $\Phi_{n-1,0}$ is $\Pi_{f}$.

(ii) The number of Jordan blocks for the eigenvalue 1 with size $n-2$ in $\Phi_{n-1,0}$ is equal to $2 \sum_{\gamma} l^{*}(\gamma)$, where $\gamma$ ranges through the compact faces of $\Gamma_{+}(f)$ such that $\operatorname{dim} \gamma=2$ and $\operatorname{rel} \operatorname{int}(\gamma) \subset \operatorname{Int}\left(\mathbb{R}_{+}^{n}\right)$. In particular, this number is even.

Note that Theorem 1.2(i) was obtained by van Doorn-Steenbrink [6] using different methods. Roughly speaking, the nilpotent part for the eigenvalue 1 in the monodromy $\Phi_{n-1,0}$ is determined by the convexity of the hypersurface $\partial \Gamma_{+}(f) \cap \operatorname{Int}\left(\mathbb{R}_{+}^{n}\right)$. Thus Theorems 1.1 and 1.2 generalize the well-known fact that the monodromies of quasi-homogeneous polynomials are semisimple. In fact, by our results in Sections 2 and 4 a general algorithm for computing all the spectral pairs of the Milnor fiber $F_{0}$ is obtained. This in particular implies that we can compute the Jordan normal form of $\Phi_{n-1,0}$ completely. Note that the spectrum of $F_{0}$ obtained by Saito [23] and Varchenko-Khovanskiu [28] is not enough to deduce the Jordan normal form. Moreover, if any compact face of $\Gamma_{+}(f)$ is prime (see Definition 2.9) we obtain also a closed formula for the Jordan normal form. See Section 4 for the details. Combining our results with those in Melle-Hernández-Torrelli-Veys [17], we can also eliminate some candidate poles of topological zeta functions.

This paper is organized as follows. In Section 2, we introduce some generalizations of the results of Danilov-Khovanskiu [3] obtained in [16]. From them we obtain a general algorithm for computing the equivariant mixed Hodge numbers of non-degenerate toric hypersurfaces. In Section 3, we recall some basic definitions and results on motivic Milnor fibers introduced by Denef-Loeser [4], [5]. Then in Section 4 , by rewriting them in terms of the Newton polyhedron $\Gamma_{+}(f)$ with the help of the results in Section 2 and [16], we prove various combinatorial formulas for the Jordan normal form of the Milnor monodromy $\Phi_{n-1,0}$. Although our proof for the eigenvalue 1 in this paper is very different from the one in [16], our results in Section 4 are completely parallel to those for monodromies at infinity obtained in [16]. We thus find a striking symmetry between local and global. For other Hodge-theoretical approaches to monodromies at infinity, see e.g. [9], [21] and [22]. Finally, let us mention that in [7] the results of this paper for the other eigenvalues $\lambda \neq 1$ have already been generalized to the monodromies over complete intersection subvarieties in $\mathbb{C}^{n}$.

\section{$\S 2$. Preliminary notions and results}

In this section, we recall the results of [16, Section 2] which will be used in this paper. They are slight generalizations of the results in Danilov-Khovanskiı [3]. 
Definition 2.1. Let $g(x)=\sum_{v \in \mathbb{Z}^{n}} a_{v} x^{v}\left(a_{v} \in \mathbb{C}\right)$ be a Laurent polynomial on $\left(\mathbb{C}^{*}\right)^{n}$.

(i) We call the convex hull of $\operatorname{supp}(g):=\left\{v \in \mathbb{Z}^{n} \mid a_{v} \neq 0\right\} \subset \mathbb{Z}^{n}$ in $\mathbb{R}^{n}$ the Newton polytope of $g$ and denote it by $\operatorname{NP}(g)$.

(ii) For $u \in\left(\mathbb{R}^{n}\right)^{*}$, we set $\Gamma(g ; u):=\left\{v \in \mathrm{NP}(g) \mid\langle u, v\rangle=\min _{w \in \mathrm{NP}(g)}\langle u, w\rangle\right\}$.

(iii) For $u \in\left(\mathbb{R}^{n}\right)^{*}$, we define the $u$-part of $g$ by $g^{u}(x):=\sum_{v \in \Gamma(g ; u)} a_{v} x^{v}$.

Definition $2.2([11])$. Let $g$ be a Laurent polynomial on $\left(\mathbb{C}^{*}\right)^{n}$. Then we say that the hypersurface $Z^{*}=\left\{x \in\left(\mathbb{C}^{*}\right)^{n} \mid g(x)=0\right\}$ of $\left(\mathbb{C}^{*}\right)^{n}$ is non-degenerate if for any $u \in\left(\mathbb{R}^{n}\right)^{*}$ the hypersurface $\left\{x \in\left(\mathbb{C}^{*}\right)^{n} \mid g^{u}(x)=0\right\}$ is smooth and reduced.

In what follows, we fix an element $\tau=\left(\tau_{1}, \ldots, \tau_{n}\right) \in T:=\left(\mathbb{C}^{*}\right)^{n}$ and let $g$ be a Laurent polynomial on $\left(\mathbb{C}^{*}\right)^{n}$ such that $Z^{*}=\left\{x \in\left(\mathbb{C}^{*}\right)^{n} \mid g(x)=0\right\}$ is non-degenerate and invariant by the automorphism $l_{\tau}:\left(\mathbb{C}^{*}\right)^{n} \underset{\tau \times}{\stackrel{\sim}{\longrightarrow}}\left(\mathbb{C}^{*}\right)^{n}$ induced by multiplication by $\tau$. Set $\Delta=\operatorname{NP}(g)$ and for simplicity assume that $\operatorname{dim} \Delta=n$. Then there exists $\beta \in \mathbb{C}$ such that $l_{\tau}^{*} g=g \circ l_{\tau}=\beta g$. This implies that for any vertex $v$ of $\Delta=\operatorname{NP}(g)$ we have $\tau^{v}=\tau_{1}^{v_{1}} \cdots \tau_{n}^{v_{n}}=\beta$. Moreover by the condition $\operatorname{dim} \Delta=n$ we see that $\tau_{1}, \ldots, \tau_{n}$ are roots of unity. For $p, q \geq 0$ and $k \geq 0$, let $h^{p, q}\left(H_{c}^{k}\left(Z^{*} ; \mathbb{C}\right)\right)$ be the mixed Hodge number of $H_{c}^{k}\left(Z^{*} ; \mathbb{C}\right)$ and set

$$
e^{p, q}\left(Z^{*}\right)=\sum_{k}(-1)^{k} h^{p, q}\left(H_{c}^{k}\left(Z^{*} ; \mathbb{C}\right)\right)
$$

as in [3]. The above automorphism of $\left(\mathbb{C}^{*}\right)^{n}$ induces a morphism of mixed Hodge structures $l_{\tau}^{*}: H_{c}^{k}\left(Z^{*} ; \mathbb{C}\right) \stackrel{\sim}{\rightarrow} H_{c}^{k}\left(Z^{*} ; \mathbb{C}\right)$ and hence $\mathbb{C}$-linear automorphisms of the $(p, q)$-parts $H_{c}^{k}\left(Z^{*} ; \mathbb{C}\right)^{p, q}$ of $H_{c}^{k}\left(Z^{*} ; \mathbb{C}\right)$. For $\alpha \in \mathbb{C}$, let $h^{p, q}\left(H_{c}^{k}\left(Z^{*} ; \mathbb{C}\right)\right)_{\alpha}$ be the dimension of the $\alpha$-eigenspace $H_{c}^{k}\left(Z^{*} ; \mathbb{C}\right)_{\alpha}^{p, q}$ of this automorphism of $H_{c}^{k}\left(Z^{*} ; \mathbb{C}\right)^{p, q}$ and set

$$
e^{p, q}\left(Z^{*}\right)_{\alpha}=\sum_{k}(-1)^{k} h^{p, q}\left(H_{c}^{k}\left(Z^{*} ; \mathbb{C}\right)\right)_{\alpha} .
$$

We call $e^{p, q}\left(Z^{*}\right)_{\alpha}$ the equivariant mixed Hodge numbers of $Z^{*}$. Since $l_{\tau}^{r}=\operatorname{id}_{Z^{*}}$ for some $r \gg 0$, these numbers are zero unless $\alpha$ is a root of unity. Obviously,

$$
e^{p, q}\left(Z^{*}\right)=\sum_{\alpha \in \mathbb{C}} e^{p, q}\left(Z^{*}\right)_{\alpha}, \quad e^{p, q}\left(Z^{*}\right)_{\alpha}=e^{q, p}\left(Z^{*}\right)_{\bar{\alpha}}
$$

In this setting, along the lines of Danilov-Khovanskiur [3] we can give an algorithm for computing these numbers $e^{p, q}\left(Z^{*}\right)_{\alpha}$ as follows. First of all, as in [3, Section 3] we have the following result. 
Proposition 2.3 ([16, Proposition 2.6]). For $p, q \geq 0$ such that $p+q>n-1$, we have

$$
e^{p, q}\left(Z^{*}\right)_{\alpha}= \begin{cases}(-1)^{n+p+1}\left(\begin{array}{c}
n \\
p+1
\end{array}\right) & (\alpha=1 \text { and } p=q), \\
0 & \text { (otherwise) }\end{cases}
$$

(we use the convention $\left(\begin{array}{l}a \\ b\end{array}\right)=0(0 \leq a<b)$ for binomial coefficients).

For a vertex $w$ of $\Delta$, consider the translated polytope $\Delta^{w}:=\Delta-w$ such that $0 \prec \Delta^{w}$ and $\tau^{v}=1$ for any vertex $v$ of $\Delta^{w}$. Then for $\alpha \in \mathbb{C}$ and $k \geq 0$ set

$$
l^{*}(k \Delta)_{\alpha}=\sharp\left\{v \in \operatorname{Int}\left(k \Delta^{w}\right) \cap \mathbb{Z}^{n} \mid \tau^{v}=\alpha\right\} \in \mathbb{Z}_{+}:=\mathbb{Z}_{\geq 0} .
$$

We can easily see that these numbers $l^{*}(k \Delta)_{\alpha}$ do not depend on the choice of the vertex $w$ of $\Delta$. We define a formal power series $P_{\alpha}(\Delta ; t)=\sum_{i \geq 0} \varphi_{\alpha, i}(\Delta) t^{i}$ by

$$
P_{\alpha}(\Delta ; t)=(1-t)^{n+1}\left\{\sum_{k \geq 0} l^{*}(k \Delta)_{\alpha} t^{k}\right\} .
$$

Then we can easily show that $P_{\alpha}(\Delta ; t)$ is actually a polynomial as in [3, Section 4.4].

Theorem 2.4 ([16, Theorem 2.7]). In the situation as above, we have

$$
\sum_{q} e^{p, q}\left(Z^{*}\right)_{\alpha}= \begin{cases}(-1)^{p+n+1}\left(\begin{array}{c}
n \\
p+1
\end{array}\right)+(-1)^{n+1} \varphi_{\alpha, n-p}(\Delta) & (\alpha=1), \\
(-1)^{n+1} \varphi_{\alpha, n-p}(\Delta) & (\alpha \neq 1) .\end{cases}
$$

By Proposition 2.3 and Theorem 2.4 we can now calculate the numbers $e^{p, q}\left(Z^{*}\right)_{\alpha}$ on the non-degenerate hypersurface $Z^{*} \subset\left(\mathbb{C}^{*}\right)^{n}$ for any $\alpha \in \mathbb{C}$ as in $\left[3\right.$, Section 5.2]. Indeed for a projective toric compactification $X$ of $\left(\mathbb{C}^{*}\right)^{n}$ such that the closure $\overline{Z^{*}}$ of $Z^{*}$ in $X$ is smooth, the variety $\overline{Z^{*}}$ is smooth projective and hence there exists a perfect pairing

$$
H^{p, q}\left(\overline{Z^{*}} ; \mathbb{C}\right)_{\alpha} \times H^{n-1-p, n-1-q}\left(\overline{Z^{*}} ; \mathbb{C}\right)_{\alpha^{-1}} \rightarrow \mathbb{C}
$$

for any $p, q \geq 0$ and $\alpha \in \mathbb{C}^{*}$ (see e.g. [29, Section 5.3.2]). Therefore, we obtain equalities $e^{p, q}\left(\overline{Z^{*}}\right)_{\alpha}=e^{n-1-p, n-1-q}\left(\overline{Z^{*}}\right)_{\alpha^{-1}}$ which are necessary to proceed with the algorithm in $[3$, Section 5.2]. We also have the following analogue of [3, Proposition 5.8].

Proposition 2.5 ([16, Proposition 2.8]). For any $\alpha \in \mathbb{C}$ and $p>0$ we have

$$
e^{p, 0}\left(Z^{*}\right)_{\alpha}=e^{0, p}\left(Z^{*}\right)_{\bar{\alpha}}=(-1)^{n-1} \sum_{\substack{\Gamma \prec \Delta \\ \operatorname{dim} \Gamma=p+1}} l^{*}(\Gamma)_{\alpha},
$$


where for a vertex $w$ of $\Gamma$ we set

$$
l^{*}(\Gamma)_{\alpha}=\sharp\left\{v \in \operatorname{Int}(\Gamma-w) \cap \mathbb{Z}^{n} \mid \tau^{v}=\alpha\right\} .
$$

The following result is an analogue of [3, Corollary 5.10]. For $\alpha \in \mathbb{C}$, denote by $\Pi(\Delta)_{\alpha}$ the number of lattice points $v=\left(v_{1}, \ldots, v_{n}\right)$ on the 1-skeleton of $\Delta^{w}=$ $\Delta-w$ such that $\tau^{v}=\alpha$, where $w$ is a vertex of $\Delta$.

Proposition 2.6 ([16, Proposition 2.9]). In the situation as above, for any $\alpha \in \mathbb{C}^{*}$ we have

$$
e^{0,0}\left(Z^{*}\right)_{\alpha}= \begin{cases}(-1)^{n-1}\left(\Pi(\Delta)_{1}-1\right) & (\alpha=1), \\ (-1)^{n-1} \Pi(\Delta)_{\alpha^{-1}} & (\alpha \neq 1) .\end{cases}
$$

For a vertex $w$ of $\Delta$, we define a closed convex cone $\operatorname{Con}(\Delta, w)$ by $\operatorname{Con}(\Delta, w)=$ $\left\{r \cdot(v-w) \mid r \in \mathbb{R}_{+}, v \in \Delta\right\} \subset \mathbb{R}^{n}$.

Definition 2.7 ([3]). Let $\Delta$ and $\Delta^{\prime}$ be two $n$-dimensional integral polytopes in $\left(\mathbb{R}^{n}, \mathbb{Z}^{n}\right)$. We denote by $\operatorname{som}(\Delta)\left(\right.$ resp. $\left.\operatorname{som}\left(\Delta^{\prime}\right)\right)$ the set of vertices of $\Delta\left(\right.$ resp. $\left.\Delta^{\prime}\right)$. Then we say that $\Delta^{\prime}$ majorizes $\Delta$ if there exists a map $\Psi: \operatorname{som}\left(\Delta^{\prime}\right) \rightarrow \operatorname{som}(\Delta)$ such that $\operatorname{Con}(\Delta, \Psi(w)) \subset \operatorname{Con}\left(\Delta^{\prime}, w\right)$ for any vertex $w$ of $\Delta^{\prime}$. For a face $\Gamma$ of $\Delta^{\prime}$ we define the face $\Psi(\Gamma)$ of $\Delta$ to be the convex hull of the points $\{\Psi(w)\}_{w \in \operatorname{som}(\Gamma)}$.

For an integral polytope $\Delta$ in $\left(\mathbb{R}^{n}, \mathbb{Z}^{n}\right)$, we denote by $X_{\Delta}$ the toric variety associated with the dual fan of $\Delta$ (see e.g. Fulton [8] and Oda [19]). Recall that if $\Delta^{\prime}$ majorizes $\Delta$ there exists a natural morphism $X_{\Delta^{\prime}} \rightarrow X_{\Delta}$.

Proposition 2.8 ([16, Proposition 2.12]). Let $\Delta$ and $Z_{\Delta}^{*}=Z^{*}$ with an action of $l_{\tau}$ be as above. Assume that an $n$-dimensional integral polytope $\Delta^{\prime}$ in $\left(\mathbb{R}^{n}, \mathbb{Z}^{n}\right)$ majorizes $\Delta$ via the map $\Psi: \operatorname{som}\left(\Delta^{\prime}\right) \rightarrow \operatorname{som}(\Delta)$. Then for the closure $\overline{Z^{*}}$ of $Z^{*}$ in $X_{\Delta^{\prime}}$ we have

$$
\begin{aligned}
\sum_{q} e^{p, q}\left(\overline{Z^{*}}\right)_{1} & =\sum_{\Gamma \prec \Delta^{\prime}}(-1)^{\operatorname{dim} \Gamma+p+1}\left\{\left(\begin{array}{c}
\operatorname{dim} \Gamma \\
p+1
\end{array}\right)-\left(\begin{array}{c}
b_{\Gamma} \\
p+1
\end{array}\right)\right\} \\
+ & \sum_{\Gamma \prec \Delta^{\prime}}(-1)^{\operatorname{dim} \Gamma+1} \sum_{i=0}^{\min \left\{b_{\Gamma}, p\right\}}\left(\begin{array}{c}
b_{\Gamma} \\
i
\end{array}\right)(-1)^{i} \varphi_{1, \operatorname{dim} \Psi(\Gamma)-p+i}(\Psi(\Gamma)),
\end{aligned}
$$

where for $\Gamma \prec \Delta^{\prime}$ we set $b_{\Gamma}=\operatorname{dim} \Gamma-\operatorname{dim} \Psi(\Gamma)$.

Definition 2.9. Let $\Delta$ be an $n$-dimensional integral polytope in $\left(\mathbb{R}^{n}, \mathbb{Z}^{n}\right)$.

(i) $([3$, Section 2.3]) We say that $\Delta$ is prime if for any vertex $w$ of $\Delta$ the cone $\operatorname{Con}(\Delta, w)$ is generated by a basis of $\mathbb{R}^{n}$. 
(ii) ([16, Definition 2.10]) We say that $\Delta$ is pseudo-prime if for any 1-dimensional face $\gamma \prec \Delta$ the number of 2-dimensional faces $\gamma^{\prime} \prec \Delta$ such that $\gamma \prec \gamma^{\prime}$ is $n-1$.

By definition, prime polytopes are pseudo-prime. Moreover any face of a pseudo-prime polytope is again pseudo-prime.

For $\alpha \in \mathbb{C} \backslash\{1\}$ and a face $\Gamma \prec \Delta$, set $\widetilde{\varphi}_{\alpha}(\Gamma)=\sum_{i=0}^{\operatorname{dim} \Gamma} \varphi_{\alpha, i}(\Gamma)$. Then as in $[3$, Section 5.5 and Theorem 5.6] we obtain the following result.

Proposition 2.10 ([16, Corollary 2.15]). Assume that $\Delta=\mathrm{NP}(g)$ is pseudoprime. Then for any $\alpha \in \mathbb{C} \backslash\{1\}$ and $r \geq 0$, we have

$$
\sum_{p+q=r} e^{p, q}\left(Z^{*}\right)_{\alpha}=(-1)^{n+r} \sum_{\substack{\Gamma \prec \Delta \\ \operatorname{dim} \Gamma=r+1}}\left\{\sum_{\Gamma^{\prime} \prec \Gamma}(-1)^{\operatorname{dim} \Gamma^{\prime}} \widetilde{\varphi}_{\alpha}\left(\Gamma^{\prime}\right)\right\} .
$$

The following lemma will be used later.

Lemma 2.11. Let $\gamma$ be a d-dimensional prime polytope. Then for any $0 \leq p \leq d$ we have

$$
\sum_{\Gamma \prec \gamma}(-1)^{\operatorname{dim} \Gamma}\left(\begin{array}{c}
\operatorname{dim} \Gamma \\
p
\end{array}\right)=\sum_{\Gamma \prec \gamma}(-1)^{d+\operatorname{dim} \Gamma}\left(\begin{array}{c}
\operatorname{dim} \Gamma \\
d-p
\end{array}\right) .
$$

Proof. For a polytope $\Delta$, denote the number of $j$-dimensional faces of $\Delta$ by $f_{\Delta, j}$ and set $f_{\Delta,-1}=1$. Let $\gamma^{\vee}$ be the dual polytope of $\gamma$. Then $\gamma^{\vee}$ is simplicial and we have $f_{\gamma^{\vee}, j}=f_{\gamma, d-1-j}$ for any $0 \leq j \leq d$. Hence (2.2) follows from the DehnSommerville equations (see e.g. [25]) for simplicial polytopes.

\section{§3. Motivic Milnor fibers}

In [4] and [5] Denef and Loeser introduced motivic Milnor fibers. In this section, we recall their definition and basic properties. Let $f \in \mathbb{C}\left[x_{1}, \ldots, x_{n}\right]$ be a polynomial such that the hypersurface $f^{-1}(0)=\left\{x \in \mathbb{C}^{n} \mid f(x)=0\right\}$ has an isolated singular point at $0 \in \mathbb{C}^{n}$. Then by a fundamental theorem of Milnor [18], for the Milnor fiber $F_{0}$ of $f$ at 0 we have $H^{j}\left(F_{0} ; \mathbb{C}\right) \simeq 0(j \neq 0, n-1)$. Denote by $\Phi_{n-1,0}: H^{n-1}\left(F_{0} ; \mathbb{C}\right) \stackrel{\sim}{\longrightarrow} H^{n-1}\left(F_{0} ; \mathbb{C}\right)$ the $(n-1)$-th Milnor monodromy of $f$ at $0 \in \mathbb{C}^{n}$. Let $\pi: X \rightarrow \mathbb{C}^{n}$ be an embedded resolution of $f^{-1}(0)$ such that $\pi^{-1}(0)$ and $\pi^{-1}\left(f^{-1}(0)\right)$ are normal crossing divisors in $X$. Let $D_{1}, \ldots, D_{m}$ be the irreducible components of $\pi^{-1}(0)$ and denote by $Z$ the proper transform of $f^{-1}(0)$ in $X$. For $1 \leq i \leq m$ denote by $a_{i}>0$ the order of the zero of $g:=f \circ \pi$ along $D_{i}$. For a non-empty subset $I \subset\{1, \ldots, m\}$ we set $d_{I}=\operatorname{gcd}\left(a_{i}\right)_{i \in I}>0, D_{I}=\bigcap_{i \in I} D_{i}$ and

$$
D_{I}^{\circ}=D_{I} \backslash\left\{\left(\bigcup_{i \notin I} D_{i}\right) \cup Z\right\} \subset X .
$$


Moreover we set

$$
Z_{I}^{\circ}=\left\{D_{I} \backslash\left(\bigcup_{i \notin I} D_{i}\right)\right\} \cap Z \subset X .
$$

Then, as in [5, Section 3.3], we can construct an unramified Galois covering $\widetilde{D_{I}^{\circ}} \rightarrow$ $D_{I}^{\circ}$ of $D_{I}^{\circ}$ as follows. First, for a point $p \in D_{I}^{\circ}$ we take an affine open neighborhood $W \subset X \backslash\left\{\left(\bigcup_{i \notin I} D_{i}\right) \cup Z\right\}$ of $p$ on which there exist regular functions $\xi_{i}(i \in I)$ such that $D_{i} \cap W=\left\{\xi_{i}=0\right\}$ for any $i \in I$. Then on $W$ we have $g=f \circ \pi=g_{1, W}\left(g_{2, W}\right)^{d_{I}}$, where we set $g_{1, W}=g \prod_{i \in I} \xi_{i}^{-a_{i}}$ and $g_{2, W}=\prod_{i \in I} \xi_{i}^{a_{i} / d_{I}}$. Note that $g_{1, W}$ is a unit on $W$ and $g_{2, W}: W \rightarrow \mathbb{C}$ is a regular function. It is easy to see that $D_{I}^{\circ}$ is covered by such affine open subsets $W$. Then as in [5, Section 3.3] by gluing the varieties

$$
\widetilde{D_{I, W}^{\circ}}=\left\{(t, z) \in \mathbb{C}^{*} \times\left(D_{I}^{\circ} \cap W\right) \mid t^{d_{I}}=\left(g_{1, W}\right)^{-1}(z)\right\}
$$

together as described below, we obtain the variety $\widetilde{D_{I}^{\circ}}$ over $D_{I}^{\circ}$. If $W^{\prime}$ is another such open subset and $g=g_{1, W^{\prime}}\left(g_{2, W^{\prime}}\right)^{d_{I}}$ is the decomposition of $g$ on it, we glue $\widetilde{D_{I, W}^{\circ}}$ and $\widetilde{D_{I, W^{\prime}}^{\circ}}$ together by the morphism $(t, z) \mapsto\left(g_{2, W^{\prime}}(z)\left(g_{2, W}\right)^{-1}(z) \cdot t, z\right)$ defined over $W \cap W^{\prime}$.

Now for $d \in \mathbb{Z}_{>0}$, let $\mu_{d} \simeq \mathbb{Z} / \mathbb{Z} d$ be the multiplicative group consisting of the $d$-roots in $\mathbb{C}$. We denote by $\hat{\mu}$ the projective limit ${\underset{\varliminf}{\longleftarrow}}_{d} \mu_{d}$ of the projective system $\left\{\mu_{i}\right\}_{i \geq 1}$ with morphisms $\mu_{i d} \rightarrow \mu_{i}$ given by $t \mapsto t^{d}$. Then the unramified Galois covering $\widetilde{D_{I}^{\circ}}$ of $D_{I}^{\circ}$ admits a natural $\mu_{d_{I}}$-action defined by assigning the automorphism $(t, z) \mapsto\left(\zeta_{d_{I}} t, z\right)$ of $\widetilde{D_{I}^{\circ}}$ to the generator $\zeta_{d_{I}}:=\exp \left(2 \pi \sqrt{-1} / d_{I}\right) \in \mu_{d_{I}}$. Thus the variety $\widetilde{D_{I}^{\circ}}$ is equipped with a good $\hat{\mu}$-action in the sense of Denef-Loeser [5, Section 2.4]. Note that also the variety $Z_{I}^{\circ}$ is equipped with the trivial good $\hat{\mu}$-action. Following the notations in [5], denote by $\mathcal{M}_{\mathbb{C}}^{\hat{\mu}}$ the ring obtained from the Grothendieck ring $\mathrm{K}_{0}^{\hat{\mu}}\left(\operatorname{Var}_{\mathbb{C}}\right)$ of varieties over $\mathbb{C}$ with good $\hat{\mu}$-actions by inverting the Lefschetz motive $\mathbb{L} \simeq \mathbb{C} \in K_{0}^{\hat{\mu}}\left(\operatorname{Var}_{\mathbb{C}}\right)$. Recall that $\mathbb{L} \in K_{0}^{\hat{\mu}}\left(\operatorname{Var}_{\mathbb{C}}\right)$ is endowed with the trivial action of $\hat{\mu}$.

Definition 3.1 (Denef and Loeser [4], [5]). We define the motivic Milnor fiber $\mathcal{S}_{f, 0} \in \mathcal{M}_{\mathbb{C}}^{\hat{\mu}}$ of $f$ at $0 \in \mathbb{C}^{n}$ by

$$
\mathcal{S}_{f, 0}=\sum_{I \neq \emptyset}\left\{(1-\mathbb{L})^{\sharp I-1}\left[\widetilde{D_{I}^{\circ}}\right]+(1-\mathbb{L})^{\sharp I}\left[Z_{I}^{\circ}\right]\right\} \in \mathcal{M}_{\mathbb{C}}^{\hat{\mu}} .
$$

As in [5, Sections 3.1.2 and 3.1.3], we denote by $\mathrm{HS}^{\text {mon }}$ the abelian category of Hodge structures with a quasi-unipotent endomorphism. Let $\mathrm{K}_{0}\left(\mathrm{HS}^{\mathrm{mon}}\right)$ be its Grothendieck ring. Then as in [5], to the cohomology groups $H^{j}\left(F_{0} ; \mathbb{C}\right)$ and the semisimple parts of their monodromy automorphisms, we can naturally associate an element

$$
\left[H_{f}\right] \in \mathrm{K}_{0}\left(\mathrm{HS}^{\mathrm{mon}}\right)
$$


To describe it in terms of $\mathcal{S}_{f, 0} \in \mathcal{M}_{\mathbb{C}}^{\hat{\mu}}$, let

$$
\chi_{h}: \mathcal{M}_{\mathbb{C}}^{\hat{\mu}} \rightarrow \mathrm{K}_{0}\left(\mathrm{HS}^{\mathrm{mon}}\right)
$$

be the Hodge characteristic morphism defined in [5] which associates to a variety $Z$ with a good $\mu_{d}$-action the Hodge structure

$$
\chi_{h}([Z])=\sum_{j \in \mathbb{Z}}(-1)^{j}\left[H_{c}^{j}(Z ; \mathbb{Q})\right] \in \mathrm{K}_{0}\left(\mathrm{HS}^{\mathrm{mon}}\right)
$$

with the action induced by $z \mapsto \exp (2 \pi \sqrt{-1} / d) z(z \in Z)$ on $Z$. Then we have the following fundamental result.

Theorem 3.2 (Denef-Loeser [4, Theorem 4.2.1]). In the Grothendieck group $\mathrm{K}_{0}\left(\mathrm{HS}^{\mathrm{mon}}\right)$, we have

$$
\left[H_{f}\right]=\chi_{h}\left(\mathcal{S}_{f, 0}\right)
$$

This theorem was proved by using the functoriality of Saito's mixed Hodge modules (see e.g. [10, Section 8.3] for a quick review of this subject).

For $\left[H_{f}\right] \in \mathrm{K}_{0}\left(\mathrm{HS}^{\mathrm{mon}}\right)$ also the following result due to Steenbrink [26] is fundamental.

Theorem 3.3 (Steenbrink [26]). In the situation as above, we have:

(i) Let $\lambda \in \mathbb{C}^{*} \backslash\{1\}$. Then $e^{p, q}\left(\left[H_{f}\right]\right)_{\lambda}=0$ for $(p, q) \notin[0, n-1] \times[0, n-1]$. Moreover for $(p, q) \in[0, n-1] \times[0, n-1]$ we have

$$
e^{p, q}\left(\left[H_{f}\right]\right)_{\lambda}=e^{n-1-q, n-1-p}\left(\left[H_{f}\right]\right)_{\lambda} .
$$

(ii) We have $e^{p, q}\left(\left[H_{f}\right]\right)_{1}=0$ for $(p, q) \notin\{(0,0)\} \sqcup([1, n-1] \times[1, n-1])$ and $e^{0,0}\left(\left[H_{f}\right]\right)_{1}=1$. Moreover for $(p, q) \in[1, n-1] \times[1, n-1]$ we have

$$
e^{p, q}\left(\left[H_{f}\right]\right)_{1}=e^{n-q, n-p}\left(\left[H_{f}\right]\right)_{1} .
$$

We can check these symmetries of $e^{p, q}\left(\left[H_{f}\right]\right)_{\lambda}$ by calculating $\chi_{h}\left(\mathcal{S}_{f, 0}\right) \in$ $\mathrm{K}_{0}\left(\mathrm{HS}^{\mathrm{mon}}\right.$ ) explicitly by our methods (see Section 4 ) in many cases. Since the weights of $\left[H_{f}\right] \in \mathrm{K}_{0}\left(\mathrm{HS}^{\mathrm{mon}}\right)$ are defined by the monodromy filtration, we have the following result.

Theorem 3.4. In the situation as above, we have:

(i) Let $\lambda \in \mathbb{C}^{*} \backslash\{1\}$ and $k \geq 1$. Then the number of Jordan blocks for the eigenvalue $\lambda$ with sizes $\geq k$ in $\Phi_{n-1,0}: H^{n-1}\left(F_{0} ; \mathbb{C}\right) \stackrel{\sim}{\longrightarrow} H^{n-1}\left(F_{0} ; \mathbb{C}\right)$ is equal to

$$
(-1)^{n-1} \sum_{p+q=n-2+k, n-1+k} e^{p, q}\left(\chi_{h}\left(\mathcal{S}_{f, 0}\right)\right)_{\lambda} .
$$


(ii) For $k \geq 1$, the number of Jordan blocks for the eigenvalue 1 with sizes $\geq k$ in $\Phi_{n-1,0}$ is equal to

$$
(-1)^{n-1} \sum_{p+q=n-1+k, n+k} e^{p, q}\left(\chi_{h}\left(\mathcal{S}_{f, 0}\right)\right)_{1} .
$$

\section{$\S 4$. Jordan normal forms of Milnor monodromies}

Our methods in [16] can also be applied to the Jordan normal forms of local Milnor monodromies. Let $f \in \mathbb{C}\left[x_{1}, \ldots, x_{n}\right]$ be a polynomial such that the hypersurface $\left\{x \in \mathbb{C}^{n} \mid f(x)=0\right\}$ has an isolated singular point at $0 \in \mathbb{C}^{n}$.

Definition 4.1. Let $f(x)=\sum_{v \in \mathbb{Z}_{+}^{n}} a_{v} x^{v} \in \mathbb{C}\left[x_{1}, \ldots, x_{n}\right]$ be a polynomial on $\mathbb{C}^{n}$.

(i) We call the convex hull of $\bigcup_{v \in \operatorname{supp}(f)}\left\{v+\mathbb{R}_{+}^{n}\right\}$ in $\mathbb{R}_{+}^{n}$ the Newton polyhedron of $f$ and denote it by $\Gamma_{+}(f)$.

(ii) The union of the compact faces of $\Gamma_{+}(f)$ is called the Newton boundary of $f$ and denoted by $\Gamma_{f}$.

(iii) We say that $f$ is convenient if $\Gamma_{+}(f)$ intersects the positive part of any coordinate axis in $\mathbb{R}^{n}$.

Definition 4.2 ([11]). We say that a polynomial $f(x)=\sum_{v \in \mathbb{Z}_{+}^{n}} a_{v} x^{v}\left(a_{v} \in \mathbb{C}\right)$ is non-degenerate at $0 \in \mathbb{C}^{n}$ if for any face $\gamma \prec \Gamma_{+}(f)$ such that $\gamma \subset \Gamma_{f}$ the complex hypersurface $\left\{x \in\left(\mathbb{C}^{*}\right)^{n} \mid f_{\gamma}(x)=0\right\}$ in $\left(\mathbb{C}^{*}\right)^{n}$ is smooth and reduced, where we set $f_{\gamma}(x)=\sum_{v \in \gamma \cap \mathbb{Z}_{+}^{n}} a_{v} x^{v}$.

Recall that generic polynomials having a fixed Newton polyhedron are nondegenerate at $0 \in \mathbb{C}^{n}$. From now on, we always assume that $f=\sum_{v \in \mathbb{Z}_{+}^{n}} a_{v} x^{v} \in$ $\mathbb{C}\left[x_{1}, \ldots, x_{n}\right]$ is convenient and non-degenerate at $0 \in \mathbb{C}^{n}$. For each face $\gamma \prec \Gamma_{+}(f)$ such that $\gamma \subset \Gamma_{f}$, let $d_{\gamma}>0$ be the lattice distance of $\gamma$ from the origin $0 \in \mathbb{R}^{n}$, and $\Delta_{\gamma}$ the convex hull of $\{0\} \sqcup \gamma$ in $\mathbb{R}^{n}$. Let $\mathbb{L}\left(\Delta_{\gamma}\right)$ be the $(\operatorname{dim} \gamma+1)$-dimensional linear subspace of $\mathbb{R}^{n}$ spanned by $\Delta_{\gamma}$ and consider the lattice $M_{\gamma}=\mathbb{Z}^{n} \cap \mathbb{L}\left(\Delta_{\gamma}\right) \simeq$ $\mathbb{Z}^{\operatorname{dim} \gamma+1}$ in it. Then we set $T_{\Delta_{\gamma}}:=\operatorname{Spec}\left(\mathbb{C}\left[M_{\gamma}\right]\right) \simeq\left(\mathbb{C}^{*}\right)^{\operatorname{dim} \gamma+1}$. Moreover let $\mathbb{L}(\gamma)$ be the smallest affine linear subspace of $\mathbb{R}^{n}$ containing $\gamma$ and for $v \in M_{\gamma}$ define their lattice heights $\operatorname{ht}(v, \gamma) \in \mathbb{Z}$ from $\mathbb{L}(\gamma)$ in $\mathbb{L}\left(\Delta_{\gamma}\right)$ so that we have ht $(0, \gamma)=$ $d_{\gamma}>0$. Then to the group homomorphism $M_{\gamma} \rightarrow \mathbb{C}^{*}$ defined by $v \mapsto \zeta_{d_{\gamma}}^{- \text {ht }(v, \gamma)}$ we can naturally associate an element $\tau_{\gamma} \in T_{\Delta_{\gamma}}$. We define a Laurent polynomial $g_{\gamma}=\sum_{v \in M_{\gamma}} b_{v} x^{v}$ on $T_{\Delta_{\gamma}}$ by

$$
b_{v}= \begin{cases}a_{v} & (v \in \gamma) \\ -1 & (v=0) \\ 0 & \text { (otherwise) }\end{cases}
$$


Then $\operatorname{NP}\left(g_{\gamma}\right)=\Delta_{\gamma}, \operatorname{supp}\left(g_{\gamma}\right) \subset\{0\} \sqcup \gamma$ and the hypersurface $Z_{\Delta_{\gamma}}^{*}=\left\{x \in T_{\Delta_{\gamma}} \mid\right.$ $\left.g_{\gamma}(x)=0\right\}$ is non-degenerate by [16, Proposition 5.3]. Moreover $Z_{\Delta_{\gamma}}^{*} \subset T_{\Delta_{\gamma}}$ is invariant under multiplication $l_{\tau_{\gamma}}: T_{\Delta_{\gamma}} \stackrel{\sim}{\longrightarrow} T_{\Delta_{\gamma}}$ by $\tau_{\gamma}$, and hence we obtain an element $\left[Z_{\Delta_{\gamma}}^{*}\right]$ of $\mathcal{M}_{\mathbb{C}}^{\hat{\mu}}$. Let $\mathbb{L}(\gamma)^{\prime} \simeq \mathbb{R}^{\operatorname{dim} \gamma}$ be a linear subspace of $\mathbb{R}^{n}$ such that $\mathbb{L}(\gamma)=\mathbb{L}(\gamma)^{\prime}+w$ for some $w \in \mathbb{Z}^{n}$ and set $\gamma^{\prime}=\gamma-w \subset \mathbb{L}(\gamma)^{\prime}$. We define a Laurent polynomial $g_{\gamma}^{\prime}=\sum_{v \in \mathbb{L}(\gamma)^{\prime} \cap \mathbb{Z}^{n}} b_{v}^{\prime} x^{v}$ on $T(\gamma):=\operatorname{Spec}\left(\mathbb{C}\left[\mathbb{L}(\gamma)^{\prime} \cap \mathbb{Z}^{n}\right]\right) \simeq\left(\mathbb{C}^{*}\right)^{\operatorname{dim} \gamma}$ by

$$
b_{v}^{\prime}= \begin{cases}a_{v+w} & \left(v \in \gamma^{\prime}\right) \\ 0 & \text { (otherwise) }\end{cases}
$$

Then $\operatorname{NP}\left(g_{\gamma}^{\prime}\right)=\gamma^{\prime}$ and the hypersurface $Z_{\gamma}^{*}=\left\{x \in T(\gamma) \mid g_{\gamma}^{\prime}(x)=0\right\}$ is nondegenerate. We define $\left[Z_{\gamma}^{*}\right] \in \mathcal{M}_{\mathbb{C}}^{\hat{\mu}}$ to be the class of the variety $Z_{\gamma}^{*}$ with the trivial action of $\hat{\mu}$. Finally let $S_{\gamma}$ be the minimal subset $S$ of $\{1, \ldots, n\}$ such that $\gamma \subset$ $\left\{\left(y_{1}, \ldots, y_{n}\right) \in \mathbb{R}^{n} \mid y_{i}=0\right.$ for any $\left.i \notin S\right\} \simeq \mathbb{R}^{\sharp S}$ and set $m_{\gamma}:=\sharp S_{\gamma}-\operatorname{dim} \gamma-1 \geq 0$. Then as in [16, Theorem 5.7] we obtain the following theorem.

Theorem 4.3. In the situation as above, we have:

(i) In the Grothendieck group $\mathrm{K}_{0}\left(\mathrm{HS}^{\mathrm{mon}}\right)$, we have

$$
\chi_{h}\left(\mathcal{S}_{f, 0}\right)=\sum_{\gamma \subset \Gamma_{f}} \chi_{h}\left((1-\mathbb{L})^{m_{\gamma}} \cdot\left[Z_{\Delta_{\gamma}}^{*}\right]\right)+\sum_{\substack{\gamma \subset \Gamma_{f} \\ \operatorname{dim} \gamma \geq 1}} \chi_{h}\left((1-\mathbb{L})^{m_{\gamma}+1} \cdot\left[Z_{\gamma}^{*}\right]\right) .
$$

(ii) Let $\lambda \in \mathbb{C}^{*} \backslash\{1\}$ and $k \geq 1$. Then the number of Jordan blocks for the eigenvalue $\lambda$ with sizes $\geq k$ in $\Phi_{n-1,0}: H^{n-1}\left(F_{0} ; \mathbb{C}\right) \stackrel{\sim}{\longrightarrow} H^{n-1}\left(F_{0} ; \mathbb{C}\right)$ is equal to

$$
(-1)^{n-1} \sum_{p+q=n-2+k, n-1+k}\left\{\sum_{\gamma \subset \Gamma_{f}} e^{p, q}\left(\chi_{h}\left((1-\mathbb{L})^{m_{\gamma}} \cdot\left[Z_{\Delta_{\gamma}}^{*}\right]\right)\right)_{\lambda}\right\} .
$$

(iii) For $k \geq 1$, the number of Jordan blocks for the eigenvalue 1 with sizes $\geq k$ in $\Phi_{n-1,0}$ is equal to

$$
\begin{aligned}
& (-1)^{n-1} \sum_{p+q=n-1+k, n+k}\left\{\sum_{\gamma \subset \Gamma_{f}} e^{p, q}\left(\chi_{h}\left((1-\mathbb{L})^{m_{\gamma}} \cdot\left[Z_{\Delta_{\gamma}}^{*}\right]\right)\right)_{1}\right. \\
& \left.+\sum_{\substack{\gamma \subset \Gamma_{f} \\
\operatorname{dim} \gamma \geq 1}} e^{p, q}\left(\chi_{h}\left((1-\mathbb{L})^{m_{\gamma}+1} \cdot\left[Z_{\gamma}^{*}\right]\right)\right)_{1}\right\} .
\end{aligned}
$$

Proof. Since (ii) and (iii) follow from (i) and Theorem 3.4, it suffices to prove (i). The proof is very similar to the one in Varchenko [27]. Let $\Sigma_{1}$ be the dual fan of $\Gamma_{+}(f)$ in $\mathbb{R}_{+}^{n}$ and $\Sigma$ its smooth subdivision. Denote by $X_{\Sigma}$ the smooth toric variety associated to $\Sigma$ (see e.g. Fulton [8] and Oda [19]). Since the union of the cones in $\Sigma$ is $\mathbb{R}_{+}^{n}$, there exists a proper morphism $\pi: X_{\Sigma} \rightarrow \mathbb{C}^{n}$. As $f$ is convenient, we can 
construct the smooth fan $\Sigma$ without subdividing the cones contained in $\partial \mathbb{R}_{+}^{n}$ (see $\left[20\right.$, Lemma (2.6), Chapter II]). Then $\pi$ induces an isomorphism $X_{\Sigma} \backslash \pi^{-1}(0) \simeq$ $\mathbb{C}^{n} \backslash\{0\}$. Moreover by the non-degeneracy at $0 \in \mathbb{C}^{n}$ of $f$, the proper transform $Z$ of the hypersurface $\left\{x \in \mathbb{C}^{n} \mid f(x)=0\right\}$ in $X_{\Sigma}$ is smooth and intersects $T$-orbits in $\pi^{-1}(0)$ transversally. Let $D_{1}, \ldots, D_{m}$ be the toric divisors in $\pi^{-1}(0) \subset X_{\Sigma}$. For a non-empty subset $I \subset\{1, \ldots, m\}$ we set $D_{I}=\bigcap_{i \in I} D_{i}$ and

$$
D_{I}^{\circ}=D_{I} \backslash\left\{\left(\bigcup_{i \notin I} D_{i}\right) \cup Z\right\} \subset X_{\Sigma}
$$

and define its unramified Galois covering $\widetilde{D_{I}^{\circ}}$ as in Section 3. Moreover we set

$$
Z_{I}^{\circ}=\left\{D_{I} \backslash\left(\bigcup_{i \notin I} D_{i}\right)\right\} \cap Z \subset X_{\Sigma}
$$

and denote by $\left[Z_{I}^{\circ}\right] \in \mathcal{M}_{\mathbb{C}}^{\hat{\mu}}$ the class of the variety $Z_{I}^{\circ}$ with the trivial action. Then, unlike the global object $\mathcal{S}_{f}^{\infty}$ in [16], Denef-Loeser's "local" motivic Milnor fiber $\mathcal{S}_{f, 0}$ contains not only $(1-\mathbb{L})^{\sharp I-1}\left[\widetilde{D_{I}^{\circ}}\right]$ but also $(1-\mathbb{L})^{\sharp I}\left[Z_{I}^{\circ}\right]$ (see Definition 3.1 ). These new elements yield the second term in the right hand side of (4.1). Finally, in the Grothendieck group $\mathrm{K}_{0}\left(\mathrm{HS}^{\mathrm{mon}}\right)$ we can rewrite $\chi_{h}\left(\mathcal{S}_{f, 0}\right)$ in terms of the dual fan $\Sigma_{1}$ (i.e. in terms of $\left.\Gamma_{+}(f)\right)$ as in the proof of [16, Theorem 5.7(i)]. This completes the proof.

Let $q_{1}, \ldots, q_{l}$ (resp. $\left.\gamma_{1}, \ldots, \gamma_{l^{\prime}}\right)$ be the 0-dimensional (resp. 1-dimensional) faces of $\Gamma_{+}(f)$ such that $q_{i} \in \operatorname{Int}\left(\mathbb{R}_{+}^{n}\right)$ (resp. $\operatorname{rel} \operatorname{int}\left(\gamma_{i}\right) \subset \operatorname{Int}\left(\mathbb{R}_{+}^{n}\right)$ ). Here rel.int $(\cdot)$ stands for the relative interior. For each $q_{i}\left(\right.$ resp. $\left.\gamma_{i}\right)$, denote by $d_{i}>0\left(\right.$ resp. $\left.e_{i}>0\right)$ the lattice distance $\operatorname{dist}\left(q_{i}, 0\right)$ (resp. $\left.\operatorname{dist}\left(\gamma_{i}, 0\right)\right)$ of it from the origin $0 \in \mathbb{R}^{n}$. For $1 \leq i \leq l^{\prime}$, let $\Delta_{i}$ be the convex hull of $\{0\} \sqcup \gamma_{i}$ in $\mathbb{R}^{n}$. Then for $\lambda \in \mathbb{C} \backslash\{1\}$ and $1 \leq i \leq l^{\prime}$ such that $\lambda^{e_{i}}=1$ we set

$$
\begin{aligned}
n(\lambda)_{i}= & \sharp\left\{v \in \mathbb{Z}^{n} \cap \operatorname{rel} . i n t\left(\Delta_{i}\right) \mid \operatorname{ht}\left(v, \gamma_{i}\right)=k\right\} \\
& +\sharp\left\{v \in \mathbb{Z}^{n} \cap \operatorname{rel} . i n t\left(\Delta_{i}\right) \mid \operatorname{ht}\left(v, \gamma_{i}\right)=e_{i}-k\right\},
\end{aligned}
$$

where $k$ is the minimal positive integer satisfying $\lambda=\zeta_{e_{i}}^{k}$ and for $v \in \mathbb{Z}^{n} \cap$ rel.int $\left(\Delta_{i}\right)$ we denote by ht $\left(v, \gamma_{i}\right)$ the lattice height of $v$ from the base $\gamma_{i}$ of $\Delta_{i}$. As in [16, Theorem 5.9], by using Propositions 2.5 and 2.6 and Theorem 4.3(ii), we obtain the following theorem.

Theorem 4.4. In the situation as above, for $\lambda \in \mathbb{C}^{*} \backslash\{1\}$, we have:

(i) The number of Jordan blocks for the eigenvalue $\lambda$ with the maximal possible size $n$ in $\Phi_{n-1,0}$ is equal to $\sharp\left\{q_{i} \mid \lambda^{d_{i}}=1\right\}$.

(ii) The number of Jordan blocks for the eigenvalue $\lambda$ with size $n-1$ in $\Phi_{n-1,0}$ is equal to $\sum_{i: \lambda^{e_{i}=1}} n(\lambda)_{i}$. 
Note that by Theorem 4.3 and our results in Section 2 we can always calculate the whole Jordan normal form of $\Phi_{n-1,0}$. Now, we shall rewrite Theorem 4.3(ii) more explicitly in the case where any face $\gamma \prec \Gamma_{+}(f)$ such that $\gamma \subset \Gamma_{f}$ is prime (see Definition 2.9(i)). Recall that by Proposition 2.3 for $\lambda \in \mathbb{C}^{*} \backslash\{1\}$ and a face $\gamma \prec \Gamma_{+}(f)$ such that $\gamma \subset \Gamma_{f}$ we have $e^{p, q}\left(Z_{\Delta_{\gamma}}^{*}\right)_{\lambda}=0$ for any $p, q \geq 0$ such that $p+q>\operatorname{dim} \Delta_{\gamma}-1=\operatorname{dim} \gamma$. So the non-negative integers $r \geq 0$ such that $\sum_{p+q=r} e^{p, q}\left(Z_{\Delta_{\gamma}}^{*}\right)_{\lambda} \neq 0$ are contained in the closed interval $[0, \operatorname{dim} \gamma] \subset \mathbb{R}$.

Definition 4.5. For a face $\gamma \prec \Gamma_{+}(f)$ such that $\gamma \subset \Gamma_{f}$ and $k \geq 1$, we define a finite subset $J_{\gamma, k} \subset[0, \operatorname{dim} \gamma] \cap \mathbb{Z}$ by

$$
J_{\gamma, k}=\{0 \leq r \leq \operatorname{dim} \gamma \mid n-2+k \equiv r \bmod 2\} .
$$

For each $r \in J_{\gamma, k}$, set

$$
d_{k, r}=\frac{n-2+k-r}{2} \in \mathbb{Z}_{+}
$$

If a face $\gamma \prec \Gamma_{+}(f)$ such that $\gamma \subset \Gamma_{f}$ is prime, then the polytope $\Delta_{\gamma}$ is pseudo-prime (see Definition 2.9(ii)). Then by Proposition 2.10 for $\lambda \in \mathbb{C}^{*} \backslash\{1\}$ and an integer $r \geq 0$ such that $r \in[0, \operatorname{dim} \gamma]$ we have

$$
\sum_{p+q=r} e^{p, q}\left(\chi_{h}\left(\left[Z_{\Delta_{\gamma}}^{*}\right]\right)\right)_{\lambda}=(-1)^{\operatorname{dim} \gamma+r+1} \sum_{\substack{\Gamma \prec \Delta_{\gamma} \\ \operatorname{dim} \Gamma=r+1}}\left\{\sum_{\Gamma^{\prime} \prec \Gamma}(-1)^{\operatorname{dim} \Gamma^{\prime}} \widetilde{\varphi}_{\lambda}\left(\Gamma^{\prime}\right)\right\} .
$$

For simplicity, we denote this last integer by $e(\gamma, \lambda)_{r}$. Then by Theorem 4.3(ii) we obtain the following result.

Theorem 4.6. Assume that any face $\gamma \prec \Gamma_{+}(f)$ such that $\gamma \subset \Gamma_{f}$ is prime. Let $\lambda \in \mathbb{C}^{*} \backslash\{1\}$ and $k \geq 1$. Then the number of Jordan blocks for the eigenvalue $\lambda$ with sizes $\geq k$ in $\Phi_{n-1,0}: H^{n-1}\left(F_{0} ; \mathbb{C}\right) \stackrel{\sim}{\longrightarrow} H^{n-1}\left(F_{0} ; \mathbb{C}\right)$ is equal to

$$
\begin{aligned}
& (-1)^{n-1} \sum_{\gamma \subset \Gamma_{f}}\left\{\sum_{r \in J_{\gamma, k}}(-1)^{d_{k, r}}\left(\begin{array}{c}
m_{\gamma} \\
d_{k, r}
\end{array}\right) \cdot e(\gamma, \lambda)_{r}\right. \\
& \left.\quad+\sum_{r \in J_{\gamma, k+1}}(-1)^{d_{k+1, r}}\left(\begin{array}{c}
m_{\gamma} \\
d_{k+1, r}
\end{array}\right) \cdot e(\gamma, \lambda)_{r}\right\},
\end{aligned}
$$

where we used the convention $\left(\begin{array}{l}a \\ b\end{array}\right)=0(0 \leq a<b)$ for binomial coefficients.

By combining the proof of [3, Theorem 5.6] and [16, Proposition 2.14] with Theorem 4.3(iii), if any face $\gamma \prec \Gamma_{+}(f)$ such that $\gamma \subset \Gamma_{f}$ is prime we can also describe the Jordan blocks for the eigenvalue 1 in $\Phi_{n-1,0}$ by a closed formula. Since this result is rather involved, we omit it here. 
We can also obtain the corresponding results for the eigenvalue 1 by rewriting Theorem 4.3(iii) more simply as follows.

Theorem 4.7. In the situation of Theorem 4.3, for $k \geq 1$ the number of Jordan blocks for the eigenvalue 1 with sizes $\geq k$ in $\Phi_{n-1,0}$ is equal to

$$
(-1)^{n-1} \sum_{p+q=n-2-k, n-1-k}\left\{\sum_{\gamma \subset \Gamma_{f}} e^{p, q}\left(\chi_{h}\left((1-\mathbb{L})^{m_{\gamma}} \cdot\left[Z_{\Delta_{\gamma}}^{*}\right]\right)\right)_{1}\right\} .
$$

This theorem will be deduced from Theorem 4.9 below. As in [16, Theorems 5.11 and 5.12], by using Propositions 2.5 and 2.6 and Theorem 4.7, we obtain the following corollary. Denote by $\Pi_{f}$ the number of lattice points on the 1-skeleton of $\Gamma_{f} \cap \operatorname{Int}\left(\mathbb{R}_{+}^{n}\right)$. Also, for a compact face $\gamma \prec \Gamma_{+}(f)$ we denote by $l^{*}(\gamma)$ the number of lattice points on rel.int $(\gamma)$.

Corollary 4.8. In the situation as above, we have:

(i) (van Doorn-Steenbrink [6]) The number of Jordan blocks for the eigenvalue 1 with the maximal possible size $n-1$ in $\Phi_{n-1,0}$ is $\Pi_{f}$.

(ii) The number of Jordan blocks for the eigenvalue 1 with size $n-2$ in $\Phi_{n-1,0}$ is equal to $2 \sum_{\gamma} l^{*}(\gamma)$, where $\gamma$ ranges through the compact faces of $\Gamma_{+}(f)$ such that $\operatorname{dim} \gamma=2$ and $\operatorname{rel} \operatorname{int}(\gamma) \subset \operatorname{Int}\left(\mathbb{R}_{+}^{n}\right)$.

Note that Corollary 4.8(i) was obtained by van Doorn-Steenbrink [6] using different methods. Theorem 4.7 asserts that by replacing $\Gamma_{+}(f)$ with the Newton polyhedron at infinity $\Gamma_{\infty}(f)$ in [13], [15] and [16] the combinatorial description of the local monodromy $\Phi_{n-1,0}$ is the same as that of the global one $\Phi_{n-1}^{\infty}$ obtained in [16, Theorem 5.7(iii)]. Thus we find a beautiful symmetry between local and global. Theorem 4.7 can be deduced from the following more precise result (use the symmetry of the numbers on the left hand side of (4.3) with respect to the line $p+q=n-2)$.

Theorem 4.9. In the situation as above, for any $0 \leq p, q \leq n-2$ we have

$$
\begin{aligned}
\sum_{\gamma \subset \Gamma_{f}} e^{p, q} & \left(\chi_{h}\left((1-\mathbb{L})^{m_{\gamma}}\left[Z_{\Delta_{\gamma}}^{*}\right]\right)\right)_{1} \\
& =\sum_{\gamma \subset \Gamma_{f}} e^{p+1, q+1}\left(\chi_{h}\left((1-\mathbb{L})^{m_{\gamma}}\left[Z_{\Delta_{\gamma}}^{*}\right]+(1-\mathbb{L})^{m_{\gamma}+1}\left[Z_{\gamma}^{*}\right]\right)\right)_{1} .
\end{aligned}
$$

We can easily see that Theorem 4.9 follows from Proposition 4.10 below. For $[V] \in \mathrm{K}_{0}\left(\mathrm{HS}^{\mathrm{mon}}\right)$, let $e([V])_{1}=\sum_{p, q=0}^{\infty} e^{p, q}([V])_{1} t_{1}^{p} t_{2}^{q}$ be the generating function of $e^{p, q}([V])_{1}$ as in [3]. 
Proposition 4.10. We have

$$
\sum_{\gamma \subset \Gamma_{f}} e\left(\chi_{h}\left((1-\mathbb{L})^{m_{\gamma}+1}\left(\left[Z_{\Delta_{\gamma}}^{*}\right]+\left[Z_{\gamma}^{*}\right]\right)\right)\right)_{1}=1-\left(t_{1} t_{2}\right)^{n}
$$

Now, we start proving Proposition 4.10. First, we apply Proposition 2.8 to the case where $\Delta=\Delta_{\gamma}$ for a face $\gamma$ of $\Gamma_{+}(f)$ such that $\gamma \subset \Gamma_{f}$. Let $\gamma^{\prime}$ be a prime polytope in $\mathbb{R}^{\operatorname{dim} \gamma}$ which majorizes $\gamma$ and consider the Minkowski sum $\gamma^{\prime \prime}:=\gamma+\gamma^{\prime}\left(\right.$ resp. $\left.\square_{\gamma^{\prime \prime}}:=\Delta_{\gamma}+\gamma^{\prime}\right)$ in $\mathbb{R}^{\operatorname{dim} \gamma}$ (resp. $\mathbb{R}^{\operatorname{dim} \gamma+1}$ ). It is well-known that any polytope has a prime polytope which majorizes it (see e.g. [2, Remark 8.3], [3] and [19]). Then $\square_{\gamma^{\prime \prime}}$ is a $(\operatorname{dim} \gamma+1)$-dimensional truncated pyramid whose top (resp. bottom) is $\gamma^{\prime}$ (resp. $\gamma^{\prime \prime}$ ) (see Figure 1 below). In particular, $\square_{\gamma^{\prime \prime}}$ is prime. Since the dual fan of $\gamma^{\prime \prime}$ coincides with that of $\gamma^{\prime}$, the prime polytope $\gamma^{\prime \prime}$ majorizes $\gamma$. Let $\Psi: \operatorname{som}\left(\gamma^{\prime \prime}\right) \rightarrow \operatorname{som}(\gamma)$ be the morphism between the sets of the vertices of $\gamma^{\prime \prime}$ and $\gamma$. By extending $\Psi$ to a morphism $\widetilde{\Psi}: \operatorname{som}\left(\square_{\gamma^{\prime \prime}}\right) \rightarrow \operatorname{som}\left(\Delta_{\gamma}\right)$ as

$$
\widetilde{\Psi}(w)= \begin{cases}\Psi(w) & \left(w \in \operatorname{som}\left(\gamma^{\prime \prime}\right)\right) \\ \{0\} & \left(w \in \operatorname{som}\left(\gamma^{\prime}\right)\right)\end{cases}
$$

we see that the prime polytope $\square_{\gamma^{\prime \prime}}$ majorizes $\Delta_{\gamma}$.

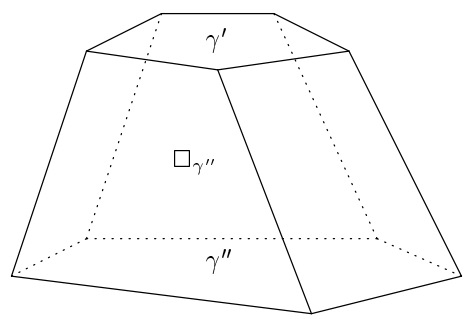

Figure 1

Proposition 4.11. For the closure $\overline{Z_{\Delta_{\gamma}}^{*}}$ of $Z_{\Delta_{\gamma}}^{*}$ in $X_{\square_{\gamma^{\prime \prime}}}$, we have

$$
\sum_{q} e^{p, q}\left(\overline{Z_{\Delta_{\gamma}}^{*}}\right)_{1}=\sum_{\tau \prec \gamma^{\prime \prime}}(-1)^{\operatorname{dim} \tau+p}\left(\begin{array}{c}
\operatorname{dim} \tau \\
p
\end{array}\right) .
$$

Proof. It suffices to rewrite Proposition 2.8 in this case. For a face $\Gamma$ of $\square_{\gamma^{\prime \prime}}$, we set $b_{\Gamma}=\operatorname{dim} \Gamma-\operatorname{dim} \widetilde{\Psi}(\Gamma)$. Note that the set of faces of $\square_{\gamma^{\prime \prime}}$ consists of those of $\gamma^{\prime}$ and $\gamma^{\prime \prime}$ and side faces. Each side face of $\square_{\gamma^{\prime \prime}}$ is a truncated pyramid $\square_{\tau}$ whose bottom is $\tau \prec \gamma^{\prime \prime}$. Since $\operatorname{dim} \square_{\tau}=\operatorname{dim} \tau+1$ and $b_{\square_{\tau}}=b_{\tau}$ for $\tau \prec \gamma^{\prime \prime}$, we have 


$$
\begin{aligned}
& \sum_{\Gamma \prec \square_{\gamma^{\prime \prime}}}(-1)^{\operatorname{dim} \Gamma+p+1}\left\{\left(\begin{array}{c}
\operatorname{dim} \Gamma \\
p+1
\end{array}\right)-\left(\begin{array}{c}
b_{\Gamma} \\
p+1
\end{array}\right)\right\} \\
&=\sum_{\tau \prec \gamma^{\prime}}(-1)^{\operatorname{dim} \tau+p+1}\left\{\left(\begin{array}{c}
\operatorname{dim} \tau \\
p+1
\end{array}\right)-\left(\begin{array}{c}
b_{\tau} \\
p+1
\end{array}\right)\right\} \\
&+\sum_{\tau \prec \gamma^{\prime \prime}}(-1)^{\operatorname{dim} \tau+p+1}\left\{\left(\begin{array}{c}
\operatorname{dim} \tau \\
p+1
\end{array}\right)-\left(\begin{array}{c}
b_{\tau} \\
p+1
\end{array}\right)\right\} \\
&+\sum_{\tau \prec \gamma^{\prime \prime}}(-1)^{\operatorname{dim} \square_{\tau}+p+1}\left\{\left(\begin{array}{c}
\operatorname{dim} \square_{\tau} \\
p+1
\end{array}\right)-\left(\begin{array}{c}
b_{\square} \\
p+1
\end{array}\right)\right\} \\
&= \sum_{\tau \prec \gamma^{\prime}}(-1)^{\operatorname{dim} \tau+p+1}\left\{\left(\begin{array}{c}
\operatorname{dim} \tau \\
p+1
\end{array}\right)-\left(\begin{array}{c}
\operatorname{dim} \tau \\
p+1
\end{array}\right)\right\} \\
&+\sum_{\tau \prec \gamma^{\prime \prime}}(-1)^{\operatorname{dim} \tau+p+1}\left\{\left(\begin{array}{c}
\operatorname{dim} \tau \\
p+1
\end{array}\right)-\left(\begin{array}{c}
b_{\tau} \\
p+1
\end{array}\right)\right\} \\
&+\sum_{\tau \prec \gamma^{\prime \prime}}(-1)^{\operatorname{dim} \tau+1+p+1}\left\{\left(\begin{array}{c}
\operatorname{dim} \tau+1 \\
p+1
\end{array}\right)-\left(\begin{array}{c}
b_{\tau} \\
p+1
\end{array}\right)\right\} \\
&= \sum_{\tau \prec \gamma^{\prime \prime}}(-1)^{\operatorname{dim} \tau+p+1}\left\{\left(\begin{array}{c}
\operatorname{dim} \tau \\
p+1
\end{array}\right)-\left(\begin{array}{c}
\operatorname{dim} \tau+1 \\
p+1
\end{array}\right)\right\} \\
&=\left.\sum_{\tau \prec \gamma^{\prime \prime}}(-1)^{\operatorname{dim} \tau+p}\left(\begin{array}{c}
\operatorname{dim} \tau \\
p
\end{array}\right)\right\}
\end{aligned}
$$

and similarly we have

$$
\begin{aligned}
\sum_{\Gamma \prec \square_{\gamma^{\prime \prime}}}(-1)^{\operatorname{dim} \Gamma+1} & \sum_{i=0}^{\min \left\{b_{\Gamma}, p\right\}}\left(\begin{array}{c}
b_{\Gamma} \\
i
\end{array}\right)(-1)^{i} \varphi_{1, \operatorname{dim} \widetilde{\Psi}(\Gamma)-p+i}(\widetilde{\Psi}(\Gamma)) \\
= & \sum_{\tau \prec \gamma^{\prime \prime}}(-1)^{\operatorname{dim} \tau+1} \sum_{i=0}^{\min \left\{b_{\tau}, p\right\}}\left(\begin{array}{c}
b_{\tau} \\
i
\end{array}\right)(-1)^{i} \\
& \times\left\{\varphi_{1, \operatorname{dim} \Psi(\tau)-p+i}(\Psi(\tau))-\varphi_{1, \operatorname{dim}} \widetilde{\Psi}\left(\square_{\tau}\right)-p+i\right.
\end{aligned}
$$

where the faces $\tau$ of the top $\gamma^{\prime}$ of $\square_{\gamma^{\prime \prime}}$ are neglected by the condition $\operatorname{dim} \widetilde{\Psi}(\tau)=0$. By $\widetilde{\Psi}\left(\square_{\tau}\right)=\Delta_{\Psi(\tau)}$ and Lemma 4.12 below, the last term is equal to 0 .

Lemma 4.12. For any face $\gamma$ of $\Gamma_{+}(f)$ such that $\gamma \subset \Gamma_{f}$, we have

$$
\varphi_{1, j+1}\left(\Delta_{\gamma}\right)=\varphi_{1, j}(\gamma)
$$

Proof. By the relation $l^{*}\left((k+1) \Delta_{\gamma}\right)_{1}-l^{*}\left(k \Delta_{\gamma}\right)_{1}=l^{*}(k \gamma)_{1}(k \geq 0)$ we have

$$
P_{1}\left(\Delta_{\gamma} ; t\right)=t P_{1}(\gamma ; t) \text {. }
$$

By comparing the coefficients of $t^{j+1}$ on both sides, we obtain (4.4). 
The following proposition is a key in the proof of Proposition 4.10.

Proposition 4.13. For any face $\gamma$ of $\Gamma_{+}(f)$ such that $\gamma \subset \Gamma_{f}$, we have

$$
e\left(\chi_{h}\left(\left[Z_{\Delta_{\gamma}}^{*}\right]+\left[Z_{\gamma}^{*}\right]\right)\right)_{1}=\left(t_{1} t_{2}-1\right)^{\operatorname{dim} \gamma} .
$$

Proof. It is enough to prove

$$
e^{p, q}\left(Z_{\gamma}^{*}\right)_{1}+e^{p, q}\left(Z_{\Delta_{\gamma}}^{*}\right)_{1}=(-1)^{\operatorname{dim} \gamma+p}\left(\begin{array}{c}
\operatorname{dim} \gamma \\
p
\end{array}\right) \cdot \delta_{p, q},
$$

where $\delta_{p, q}$ is Kronecker's delta. We consider the closure $\overline{Z_{\Delta_{\gamma}}^{*}}$ of $Z_{\Delta_{\gamma}}^{*}$ in $X_{\square_{\gamma^{\prime \prime}}}$. Then by the proofs of Propositions 2.8 and 4.11 (i.e. by the decomposition of the toric variety $X_{\square_{\gamma^{\prime \prime}}}$ into $T$-orbits), we have

$$
\begin{aligned}
& e^{p, q}\left(\overline{Z_{\Delta_{\gamma}}^{*}}\right)_{1}=\sum_{\tau \prec \gamma^{\prime \prime}}\left\{e^{p, q}\left(\left(\mathbb{C}^{*}\right)^{b_{\tau}} \times Z_{\Psi(\tau)}^{*}\right)_{1}+e^{p, q}\left(\left(\mathbb{C}^{*}\right)^{b_{\tau}} \times Z_{\widetilde{\Psi}\left(\square_{\tau}\right)}^{*}\right)_{1}\right\} \\
& \quad=\sum_{\tau \prec \gamma^{\prime \prime}} \sum_{i=0}^{\min \left\{b_{\tau}, p\right\}}\left(\begin{array}{c}
b_{\tau} \\
i
\end{array}\right)(-1)^{i+b_{\tau}}\left\{e^{p-i, q-i}\left(Z_{\Psi(\tau)}^{*}\right)_{1}+e^{p-i, q-i}\left(Z_{\Delta_{\Psi(\tau)}}^{*}\right)_{1}\right\} .
\end{aligned}
$$

Let us prove (4.5) by induction on $\operatorname{dim} \gamma$. In the case $\operatorname{dim} \gamma=0,(4.5)$ follows easily from Propositions 2.3 and 2.6. Assume that (4.5) holds for any $\sigma \subset \Gamma_{f}$ such that $\operatorname{dim} \sigma<\operatorname{dim} \gamma$. Then by $b_{\gamma^{\prime \prime}}=0$ and (4.6) we have

$$
e^{p, q}\left(\overline{Z_{\Delta_{\gamma}}^{*}}\right)_{1}=e^{p, q}\left(Z_{\gamma}^{*}\right)_{1}+e^{p, q}\left(Z_{\Delta_{\gamma}}^{*}\right)_{1}+\delta_{p, q} \sum_{\tau \supsetneqq \gamma^{\prime \prime}}(-1)^{\operatorname{dim} \tau+p}\left(\begin{array}{c}
\operatorname{dim} \tau \\
p
\end{array}\right) .
$$

In the case $p+q>\operatorname{dim} \gamma$, by Proposition 2.3 we have

$$
e^{p, q}\left(\overline{Z_{\Delta_{\gamma}}^{*}}\right)_{1}=\delta_{p, q} \sum_{\tau \prec \gamma^{\prime \prime}}(-1)^{\operatorname{dim} \tau+p}\left(\begin{array}{c}
\operatorname{dim} \tau \\
p
\end{array}\right) .
$$

Therefore, also in the case $p+q<\operatorname{dim} \gamma$, by the Poincaré duality for $\overline{Z_{\Delta_{\gamma}}^{*}}\left(\square_{\gamma^{\prime \prime}}\right.$ is prime) and Lemma 2.11 we have

$$
\begin{aligned}
e^{p, q}\left(\overline{Z_{\Delta_{\gamma}}^{*}}\right)_{1} & =e^{\operatorname{dim} \gamma-p, \operatorname{dim} \gamma-q}\left(\overline{Z_{\Delta_{\gamma}}^{*}}\right)_{1}=\delta_{p, q} \sum_{\tau \prec \gamma^{\prime \prime}}(-1)^{\operatorname{dim} \tau+\operatorname{dim} \gamma-p}\left(\begin{array}{c}
\operatorname{dim} \tau \\
\operatorname{dim} \gamma-p
\end{array}\right) \\
& =\delta_{p, q} \sum_{\tau \prec \gamma^{\prime \prime}}(-1)^{\operatorname{dim} \tau+p}\left(\begin{array}{c}
\operatorname{dim} \tau \\
p
\end{array}\right) .
\end{aligned}
$$

In the case $p+q=\operatorname{dim} \gamma$, by Proposition 4.11 and the previous results we have

$e^{p, q}\left(\overline{Z_{\Delta_{\gamma}}^{*}}\right)_{1}=\sum_{q^{\prime}} e^{p, q^{\prime}}\left(\overline{Z_{\Delta_{\gamma}}^{*}}\right)_{1}-\left(1-\delta_{p, q}\right) e^{p, p}\left(\overline{Z_{\Delta_{\gamma}}^{*}}\right)_{1}=\delta_{p, q} \sum_{\tau \prec \gamma^{\prime \prime}}(-1)^{\operatorname{dim} \tau+p}\left(\begin{array}{c}\operatorname{dim} \tau \\ p\end{array}\right)$.

By (4.7), we obtain (4.5) for any $p, q$. 
Now we can finish the proof of Proposition 4.10 as follows. By Proposition 4.13 , we have

$$
\begin{gathered}
\sum_{\gamma \subset \Gamma_{f}} e\left(\chi_{h}\left((1-\mathbb{L})^{m_{\gamma}+1}\left(\left[Z_{\Delta_{\gamma}}^{*}\right]+\left[Z_{\gamma}^{*}\right]\right)\right)\right)_{1}=\sum_{\gamma \subset \Gamma_{f}}\left(1-t_{1} t_{2}\right)^{m_{\gamma}+1}\left(t_{1} t_{2}-1\right)^{\operatorname{dim} \gamma} \\
=\sum_{l=1}^{n}\left(1-t_{1} t_{2}\right)^{l} \sum_{\sharp S_{\gamma}=l}(-1)^{\operatorname{dim} \gamma}=\sum_{l=1}^{n}\left(1-t_{1} t_{2}\right)^{l}\left(\begin{array}{c}
n \\
l
\end{array}\right)(-1)^{l-1}=1-\left(t_{1} t_{2}\right)^{n} .
\end{gathered}
$$

Remark 4.14. Following the proof of [16, Theorem 5.16], we can easily give another proof of the Steenbrink conjecture which was proved by Varchenko-Khovanskiı [28] and Saito [23] independently. For an introduction to this conjecture, see an excellent survey by Kulikov [12].

Remark 4.15. For a polynomial map $f: \mathbb{C}^{n} \rightarrow \mathbb{C}$, it is well-known that there exists a finite subset $B \subset \mathbb{C}$ such that the restriction

$$
\mathbb{C}^{n} \backslash f^{-1}(B) \rightarrow \mathbb{C} \backslash B
$$

of $f$ is a locally trivial fibration. We denote by $B_{f}$ the smallest such subset $B \subset \mathbb{C}$. For a point $b \in B_{f}$, take a small circle $C_{\varepsilon}(b)=\{x \in \mathbb{C}|| x-b \mid=\varepsilon\}(0<\varepsilon \ll 1)$ around $b$ such that $B_{f} \cap\{x \in \mathbb{C}|| x-b \mid \leq \varepsilon\}=\{b\}$. Then by the restriction of $\mathbb{C}^{n} \backslash f^{-1}\left(B_{f}\right) \rightarrow \mathbb{C} \backslash B_{f}$ to $C_{\varepsilon}(b) \subset \mathbb{C} \backslash B_{f}$ we obtain a geometric monodromy automorphism $\Phi_{f}^{b}: f^{-1}(b+\varepsilon) \stackrel{\sim}{\rightarrow} f^{-1}(b+\varepsilon)$ and the associated linear maps

$$
\Phi_{j}^{b}: H^{j}\left(f^{-1}(b+\varepsilon) ; \mathbb{C}\right) \stackrel{\sim}{\rightarrow} H^{j}\left(f^{-1}(b+\varepsilon) ; \mathbb{C}\right) \quad(j=0,1, \ldots) .
$$

The eigenvalues of $\Phi_{j}^{b}$ were studied in [15, Sections 3 and 4]. If $f$ is tame at infinity, as in [16, Section 4] we can introduce a motivic Milnor fiber $\mathcal{S}_{f}^{b} \in \mathcal{M}_{\mathbb{C}}^{\hat{\mu}}$ along the central fiber $f^{-1}(b)$ to calculate the numbers of Jordan blocks for the eigenvalues $\lambda \neq 1$ in $\Phi_{n-1}^{b}$. This result can be easily obtained by using the proof of Sabbah [22, Theorem 13.1]. It is an interesting problem to construct a motivic object to calculate the eigenvalue 1 part of $\Phi_{n-1}^{b}$.

\section{References}

[1] N. A'Campo, La fonction zêta d'une monodromie, Comment. Math. Helv. 50 (1975), 233248. Zbl 0333.14008 MR 0371889

[2] V. I. Danilov, The geometry of toric varieties, Russian Math. Surveys 33 (1978), no. 2, 97-154. Zbl 0425.14013 MR 0495499

[3] V. I. Danilov and A. G. Khovanskiǔ, Newton polyhedra and an algorithm for computing Hodge-Deligne numbers, Math. USSR-Izv. 29 (1987), 279-298. Zbl 0669.14012 MR 0873655 
[4] J. Denef and F. Loeser, Motivic Igusa zeta functions, J. Algebraic Geom. 7 (1998), 505-537. Zbl 0943.14010 MR 1618144

[5] Geometry on arc spaces of algebraic varieties, in European Congress of Mathematics, Vol. I (Barcelona, 2000), Progr. Math. 201, Birkhäuser, 2001, 327-348. Zbl 1079.14003 MR 1905328

[6] M. G. M. van Doorn and J. H. M. Steenbrink, A supplement to the monodromy theorem, Abh. Math. Sem. Univ. Hamburg 59 (1989), 225-233. Z Zbl 0712.32022 MR 1049897

[7] A. Esterov and K. Takeuchi, Motivic Milnor fibers over complete intersection varieties and their virtual Betti numbers, Int. Math. Res. Notices 2012, 3567-3613. Zbl 1250.32025 MR 2959042

[8] W. Fulton, Introduction to toric varieties, Princeton Univ. Press, 1993. Zbl 0813.14039 MR 1234037

[9] R. García López and A. Némethi, Hodge numbers attached to a polynomial map, Ann. Inst. Fourier (Grenoble) 49 (1999), 1547-1579. Zbl :0944.32029 MR 1723826

[10] R. Hotta, K. Takeuchi and T. Tanisaki, D-modules, perverse sheaves and representation theory, Birkhäuser Boston, 2008. Zbl 1136.14009 MR 2357361

[11] A. G. Kouchnirenko, Polyèdres de Newton et nombres de Milnor, Invent. Math. 32 (1976), 1-31. Zbl 0328.32007

[12] V. S. Kulikov, Mixed Hodge structures and singularities, Cambridge Univ. Press, 1998. Zbl 0902.14005 MR 1621831

[13] A. Libgober and S. Sperber, On the zeta function of monodromy of a polynomial map, Compos. Math. 95 (1995), 287-307. Zbl 0911.11030 MR 1386922

[14] Y. Matsui and K. Takeuchi, Milnor fibers over singular toric varieties and nearby cycle sheaves, Tohoku Math. J. 63 (2011), 113-136. Zbl 1223.32019 MR 2788778

[15] - Monodromy zeta functions at infinity, Newton polyhedra and constructible sheaves, Math. Z. 268 (2011), 409-439. Zbl 1264.14005 MR 2805442

[16] Monodromy at infinity of polynomial maps and Newton polyhedra (with appendix by C. Sabbah), Int. Math. Res. Notices 2013, 1691-1746. MR 3047486

[17] A. Melle-Hernández, T. Torrelli and W. Veys, On 'maximal' poles of zeta functions, roots of $b$-functions and monodromy Jordan blocks, J. Topol. 2 (2009), 517-526. Zbl 1208.14003 MR 2546584

[18] J. Milnor, Singular points of complex hypersurfaces, Princeton Univ. Press, 1968. Zbl 0184.48405 MR 0239612

[19] T. Oda, Convex bodies and algebraic geometry. An introduction to the theory of toric varieties, Springer, 1988. Zbl 0628.52002 MR 0922894

[20] M. Oka, Non-degenerate complete intersection singularity, Hermann, Paris, 1997. Zbl 0930.14034 MR 1483897

[21] M. Raibaut, Fibre de Milnor motivique à l'infini, C. R. Math. Acad. Sci. Paris 348 (2010), 419-422. Zbl 1195.14028 MR 2607031

[22] C. Sabbah, Hypergeometric periods for a tame polynomial, Port. Math. 63 (2006), 173-226. Zbl 1113.14011 MR 2229875

[23] M. Saito, Exponents and Newton polyhedra of isolated hypersurface singularities, Math. Ann. 281 (1988), 411-417. Zbl 0628.32038 MR 0954149

[24] M. Schulze, Algorithms for the Gauss-Manin connection, J. Symbolic Comput. 32 (2001), 549-564. Zbl 0987.32014 MR 1858010

[25] D. M. Y. Sommerville, The relations connecting the angle-sums and volume of a polytope in space of $n$ dimensions, Proc. Roy. Soc. London Ser. A 115 (1927), 103-119. JFM 53.0578.03 
[26] J. H. M. Steenbrink, Mixed Hodge structures on the vanishing cohomology, in Real and complex singularities, Sijthoff and Noordhoff, Alphen aan den Rijn, 1977, 525-563. Zbl 0373.14007 MR 0485870

[27] A. N. Varchenko, Zeta-function of monodromy and Newton's diagram, Invent. Math. 37 (1976), 253-262. Zbl 0333.14007 MR 0424806

[28] A. N. Varchenko and A. G. Khovanskiü, Asymptotic behavior of integrals over vanishing cycles and the Newton polyhedron, Dokl. Akad. Nauk SSSR 283 (1985), 521-525 (in Russian). Zbl 0595.32012 MR 0800892

[29] C. Voisin, Hodge theory and complex algebraic geometry, I, Cambridge Univ. Press, 2007. Zbl 1129.14019 MR 2451566 